\title{
HEGEL Y SU TEORÍA CRÍTICA DEL DERECHO: LA POSIBILIDAD DE UNA LECTURA PRAGMATISTA
}

\author{
Cristobal Balbontin ${ }^{1}$ \\ Universidad Austral de Chile \\ cbalbonting@gmail.com
}

\begin{abstract}
RESUMEN / ABSTRACT
El presente ensayo se fija como propósito precisar la crítica hegeliana del Derecho natural y del Derecho abstracto y determinar una doctrina propiamente hegeliana del Derecho. En este sentido, nuestra hipótesis es que es en el contexto de la doctrina de la Sittlichkeitque el Derecho debe ser comprendido según Hegel. En efecto, en sus Lecciones sobre la filosofia de la historia, Hegel define la Sittlichkeit como un "orden de lo sustancial (...) de suerte que todo sujeto singular tiene la universalidad por propósito, por espíritu y por costumbre ética, y que es en y por este espíritu que él quiere, actúa, vive y goza, si bien que es su naturaleza, a saber su naturaleza segunda y espiritual"'. Luego para Hegel la normatividad jurídica debe ser comprendida al interior de este campo, en un sentido social más amplio de normatividad constituido por la praxis de una comunidad que configura un campo inmanente de objetividad que estructura cotidianamente la vida de los individuos. Lejos entonces de una separación de deber y ser, Hegel nos llama a comprender la forma cómo la norma está presente en el corazón del mundo de la vida como reconciliación de lo racional con lo real en la efectividad.
\end{abstract}

Palabras Clave: Derecho positivo, Derecho Natural, praxis, Sittlichkeit, contexto.

\section{HEGEL AND HIS CRITICAL THEORY OF RIGHT}

The present essay intends to precise a Hegel's critic of Natural Law and of Positive Law and to determine, hence, a proper Hegelian doctrine of Law. In this sense, our hypothesis is that it is in the context of the Sittlichkeit doctrine, that Law must be understood according to Hegel. Namely, in his Lessons on the philosophy of history, Hegel defines Sittlichkeit like an "order of the substantial thing (...) in such a way that all singular subject has the universality by intention, spirit and ethical custom, and that is in and by this spirit which it wants, it acts, it lives and it enjoys, even though it is his nature, that is to say his nature second and spiritual one". Therefore Hegel understands that legal normativity must be included and be understood in a wide field, constituted by the praxis of a community that forms an immanent field of objectivity that daily structure

1 Profesor auxiliar Universidad Austral de Chile, Helena Haverbeck s/n, Campus Isla Teja, Valdivia, Chile. Este escrito forma parte del proyecto FONDECYT Iniciación No 11190725.

2 Hegel, Vorlesungen über die Geschichte der Philosophie, Werke in zwanzig Bänden, ed. de E. Moldenhauer y K.M. Michel, Surkhamp Verlag, Frankfurt, 1969-1971, Bd. 19, pp. 113-114. 
the life of individuals. Far from a separation between duty and being, Hegel calls us to understand the form how the norm is present in the heart of the world of the life like a reconciliation of the rational with the real in the form of effectiveness.

KEYWORDS: Positive Law, Natural Law, praxis, Sittlichkeit, context.

$\mathbb{R A}$ La anécdota es conocida. Un día de verano, concretamente el 14 de julio de 1793, Hegel, Schelling y Hölderlin -compañeros de seminario en Tübingense reunieron secretamente a plantar un "árbol de la libertad", cantaron la Marsellesa y bailaron el Carmagnola (Valenzuela 2006, p. 271). Pero la amistad que los unía no era puramente circunstancial, sino que estaba cruzada por la fascinación juvenil ante los ideales republicanos de la Revolución francesa, en un sentido bien preciso. En efecto, tanto Schelling, como Hegel y Hölderlin, vieron en los acontecimientos revolucionarios de 1789 la posibilidad de retorno de la bella totalidad helénica: $\tilde{c} v \kappa \alpha \grave{~}$ $\pi \tilde{\alpha} v$, “uno y todo". Antes que un aforismo romántico, la frase resume una preocupación especulativa por recuperar la unidad del mundo antiguo fracturado por el solipsismo individualista de la modernidad. Sin embargo, para el joven Hegel tal preocupación debía concretarse, ante todo, en una sistematización racional del campo ético-político; sistematización en la cual el Derecho ocupa un lugar preferente dentro del orden de sus preocupaciones. En este sentido, nuestra posición es que siendo Hegel igualmente crítico con las doctrinas del Derecho positivo y con las doctrinas del Derecho natural, no obstante él hace suyas ciertas premisas de ambas doctrinas para sobrepasarlas en una tercera vía. Sostenemos que Hegel se propone amplificar los motivos sensibles de la vida ética en el Derecho, entendido como campo de reconciliación entre la vida y la razón. En este sentido, el Derecho debe ser entendido como un momento al interior de la normatividad de la Sittlichkeit. Porque, si bien Hegel reconoce el carácter propio y una particularidad científica que debe reservarse a la esfera jurídica, esta esfera no puede ser hipostasiada, o comprendida al margen de las distintas manifestaciones normativas que comprende el conjunto de la vida social. Desde este punto de vista, el Derecho es una forma social entre formas sociales que caracterizan el conjunto de la cultura.

\section{Crítica del Derecho positivo, crítica del Derecho natural}

Tempranamente -en los escritos teológicos de Frankfurt- Hegel centra sus inquietudes en denunciar en el judaísmo el culto a un Dios ausente, lo que se traduce en la sumisión a un legalismo positivo y abstracto. Culto a la letra de la ley "revelada" como reverso inevitable de la teología negativa que inspira la religión de Abraham. Así, la religión judía para Hegel es una religión que descansa en un principio de heteronomía y exterioridad de la ley con respecto al mundo, a partir de una escisión fundamental entre los hombres y la naturaleza (1907). Más tarde, en el período de Jena, los términos de la crítica varían, pero los motivos en contra de lo que Hegel llama "el Derecho abstracto" se mantienen. Concretamente, en Über die wissenschaftlichen Behandlungsarten des 
Naturrechts ${ }^{3}$, Hegel critica el formalismo exterior y arbitrario de la doctrina del Derecho positivo, señalando que al absolutizar la idealidad universal, el racionalismo establece como principio de la universalidad pura una cierta idea de la razón que no se puede realizar adecuadamente pues ella se construye-precisamente-a partir de una oposición con la naturaleza sensible. Escribe Hegel: "El formalismo, desde que ha pasado de un contenido a otro por pura negación, no puede (...) llegar a nada distinto que a relaciones o identidades relativas" (1967, p. 16). Tales reservas frente al formalismo jurídico se confirman en el conjunto de manuscritos denominados por Karl Rosenkranz el Sistema de la Vida Ética, donde Hegel explica el crimen como una revuelta del delincuente en contra de una identidad jurídica puramente formal, con la consecuente exclusión de la diferencia empírica que lo caracteriza al interior de un movimiento de lucha por el reconocimiento más amplio de su personalidad. A su vez, tanto en la Fenomenología del espíritu como en las Lecciones de la filosofía de la historia se mantienen las reservas de Hegel anteriormente señaladas en la parte consagrada al Derecho romano, donde describe un cuadro particularmente severo del formalismo jurídico.

Por su parte, en la Enciclopedia, Hegel habla, incluso, de "derecho jurídicamente limitado" para referirse al formalismo jurídico propio de la doctrina del Derecho positivo (1986, §486). Así, el problema del Derecho positivo para Hegel parece radicar en que su normatividad permanece inmanente al campo de objetividad como solidaria de la separación de Sollen y Sein, la que más tarde según Kelsen -en la Teoría Pura del Derecho-debe ser asumida como condición de posibilidad del Derecho. Es precisamente este tipo de razonamientos el que se encuentra tras la tesis de la autosuficiencia de un sistema jurídico, tal como es defendida por los teóricos del Derecho positivo ${ }^{4}$. De este modo, en la conceptualización jurídica de la persona, la propiedad, el contrato, entre otras nociones puestas a la obra por el ordenamiento jurídico, se definen esquemas racionales de objetivación cada vez más formales, que terminan sacrificando el carácter concreto de las instituciones ético-políticas y la singularidad material de un mundo donde -finalmente- el Derecho debe operar. ¿Acaso la objetivación de la declaración de la voluntad soberana en la ley no presupone como condición de su efectuación una voluntad subjetiva, en la que el Derecho se pueda realizar efectivamente? Así, la doctrina del Derecho positivo, para Hegel, es violencia, al decretar la servidumbre de lo vivo a la dominación de la ley, como si se compareciese ante ella como ante una autoridad -a la vez- extraña y extranjera.

3 Über die wissenschaftlichen Behandlungsarten des Naturrechts, seine Stelle in der praktischen Philosophie und sein Verhältnis zu den positiven Rechtswissenschaften, aparecido en 18021803 en el Kritisches Journal der Philosophie, editado y publicado ese año por Hegel y Schelling.

4 Para Kelsen la teoría de la norma fundamental tiene precisamente como propósito dar un fundamento teórico a la tesis positivista del cierre del orden normativo. Señala Kelsen: "Todas las normas - cuya validez puede ser asociada a una sola y misma norma fundamentalforman un sistema de normas, un orden normativo (...). Es esta norma fundamental la que funda la unidad de una pluralidad de normas". Kelsen, Hans, Teoría pura del Derecho, Ciudad de Mexico: Porrúa, 1993, pp. 194-195. 
Invirtiendo los motivos, pero no su sentido crítico, Hegel ataca igualmente la doctrina del Derecho natural. En Glauben und Wissen, en la Differenzschrift, pero sobre todo en su artículo sobre el Derecho natural, Hegel se encarga de denunciar aquella doctrina donde "la singularidad es erigida en objetivo y esencia de todo" (1967, pp. 17-18). Esta determinación del Derecho natural es de este modo solidaria de un empirismo que fija una identidad inmediata del individuo a partir de una diferencia relativa, impidiendo el pasaje a una identidad racional objetiva común a cada particularidad. Ya sea en la identidad inmediata del individuo, concretamente en el instinto de conservación como lo expone Rousseau (1821), ya sea en la relación positiva con los otros en el amor en Pufendorf (1989), o en la relación negativa con los otros a través del odio en Hobbes (2000), es la sensibilidad individual inmediata el elemento predominante. Ello es así ya que "cada particularidad tiene derechos iguales a los de otra particularidad (...) cada una de estas determinaciones [particulares] es tan real como la otra" (Hegel 1967, p. 20). De este modo, el empirismo excluye de ella toda identidad verdadera, porque esa identidad es puramente inmediata del individuo, con lo cual el empirismo imposibilita el pasaje de la multiplicidad de individualidades particulares a una identidad objetiva común en el Derecho. Es por ello que en la teoría del contrato social en Hobbes o Rousseau el pasaje al estado civil implica que las particularidades individuales sean englobadas por el Estado en una unidad puramente exterior. Tal es el campo de una doctrina empirista del Derecho natural que -en los términos del Sistema de la Vida Ética de 1802- está dominada por una intuición sin conceptos.

En este sentido, la doctrina del Derecho natural racional de Kant también produce efectivamente este principio de identidad, pero de una forma puramente racional. Dicho de otro modo, se trata en Kant de una identidad que se cauciona por la negación de toda forma empírica de la sensibilidad. De esta forma, el Derecho natural racional de Kant expresa una preocupación puramente represiva de lo sensible por lo inteligible. Así, la filosofía kantiana cae en una contradicción, pues el legislador universal es un individuo, una finitud empírica de la cual sería solidaria la universalidad que precisamente se pretende establecer. De este modo, el idealismo subjetivo de Kant niega en su formalismo el empirismo; pero, al mismo tiempo, opera una formulación incompleta de la universalidad normativa, pues ella toma por base una finitud que define un gesto que se organiza en una oposición recíproca entre lo finito y lo infinito, entre sensibilidad y entendimiento, sin reconciliación posible; lo que implica la más aguda de las contradicciones. Como Hegel lo resume bien: "Cada término es una cosa que no es la otra" (1967, p. 93). Así, la metafísica de la subjetividad y el concepto del entendimiento terminan por construir una economía violenta del Derecho pues -como señala Hegel-, "él nace de un tormento que no tiene fin, para encontrar una relación necesaria de lo uno a lo otro y la dominación necesaria de los unos sobre los otros" (1967, p. 18). Consecuentemente, Hegel escribe en su artículo Glauben und Wissen que "es conocido que a lo empírico, el concepto infinito le es absolutamente opuesto, y que la esfera de esta oposición - una finitud y una infinitud- es absoluta" (1968, p. 322). En dichas circunstancias, es dable comprender que para Kant el Derecho signifique antes que nada una limitación o una restricción del libre arbitrio, de tal manera que puedan coexistir "la libertad con la libertad de todo otro individuo, según una ley universal" 
(Kant 1968, p. 231). Sin embargo, para Hegel, tal comprensión del Derecho termina por consolidar un error filosófico, pues ella hace derivar la voluntad racional de la voluntad del individuo singular "en el arbitrio (Willkür) que le es propio" (Hegel 1950, §29). En síntesis, al presuponer la exterioridad recíproca del universal y de la singularidad, del Derecho y de los derechos, Kant no puede ver en el Derecho objetivo más que una pura limitación de los derechos subjetivos. Luego, en Kant-según Hegel-los derechos impiden un genuino pasaje al Derecho.

\section{La vía pragmatista}

Contra las doctrinas del Derecho positivo y del Derecho natural, pero a la vez haciendo suyas premisas de ambas doctrinas, nuestra tesis es que Hegel se propone amplificar los motivos sensibles de la vida ética en el Derecho como campo de reconciliación posible de la vida y la razón. Aun cuando -desde la Fenomenología del espíritu en adelante- Hegel se distancia de Schelling para indicarnos que el espíritu es más alto que la naturaleza. Pero, el proyecto hegeliano no busca desprenderse de la dimensión de la vida sensible sino darle continuidad y realización en el espíritu. Desde este punto de vista, Hegel no abandona la perspectiva empirista del Derecho natural tal como la desarrolla Rousseau, sino que pretende corregirla, ampliando sus motivos a una genuina dimensión objetiva.

En efecto, retomando el tema spinozista de una potencia que produce y se reproduce por sus accidentes (Spinoza 2005, I proposición 10), la empresa hegeliana consiste en describir el itinerario productivo del espíritu ético hasta su culminación en el Derecho. De hecho, Hegel define el Derecho como “objetivación del espíritu” (1950, $\S 30)$. De este modo, Hegel busca tomar del empirismo una cierta figura práctica del eudaimonismo antiguo, que privilegia el contenido ético existente en el sentimiento. Los motivos del romanticismo -como los lazos éticos constituidos por el amor y la familia- son conservados, a la vez que Hegel toma de Kant la necesidad de penetrar en la dimensión objetiva del Derecho como instauración racional de una identidad ética de carácter universal. De este modo para Hegel la vida ética verdadera es la vida de los individuos en y por la totalidad ética, tal como Hegel la comprende a propósito del $\pi o \lambda \imath \tau \varepsilon v ́ \varepsilon \imath v$ antiguo (Bourgeois 1986, p. 501).

Tal es -en lo fundamental- la génesis de la doctrina de la Sittlichkeit, en cuyo contexto el Derecho debe ser comprendido. En efecto, en sus Lecciones sobre la filosofia de la historia, Hegel define la Sittlichkeit como un "orden de lo sustancial (...) de suerte que todo sujeto singular tiene la universalidad por propósito, por espíritu y por costumbre ética, y que es en y por este espíritu que él quiere, actúa, vive y goza, si bien que es su naturaleza, a saber su naturaleza segunda y espiritual" (1969-1971, pp. 113-114). Luego, de acuerdo con lo precedente, para Hegel la normatividad jurídica debe ser comprendida al interior de este campo, es decir, según su sentido social más amplio de normatividad constituido por los usos, costumbres y praxis de una comunidad que configura un campo inmanente de objetividad que estructura cotidianamente la vida de los individuos y al cual -comúnmente- nos referimos bajo la denominación 
de cultura. Lejos entonces de una separación de deber y ser, de teoría y praxis, Hegel nos llama a comprender la forma cómo la norma está presente en el corazón del mundo de la vida como reconciliación de lo racional con lo real en la efectividad. De hecho, cuando Hegel publica en 1820 los Principios de la filosofía del derecho, el texto está desarrollado tomando por base la Enciclopedia, del cual su primera versión contemplaba como segundo título Naturrecht und Staatswissenschaft ("Derecho natural y ciencia del Estado"), título destinado a relativizar el derecho en sí y asociarlo a un contexto extra-jurídico. Asimismo, en los cursos de 1817-1818, Hegel prefiere abandonar el término "Derecho natural" en favor de "la doctrina filosófica del Derecho o del espíritu objetivo" (Hegel 1955, p.21), lo que demuestra la necesidad de relacionar el Derecho con un contexto extrajurídico al interior del cual él se entronca. En este sentido es relevante la precisión que Hegel realiza en los Principios de la filosofía del derecho, señalando que "la ciencia filosófica del Derecho tiene por objeto la idea del Derecho, a saber [no sólo] el concepto del Derecho sino la efectuación de éste" (1950, §1). Es decir, el problema del Derecho no implica solo su formulación intelectual, sino que es indisociable de su efectuación extra-jurídica. Así, los conceptos jurídicos no son receptáculos muertos, sino que deben ser comprendidos a partir del espíritu vivo de lo efectivo. Y por esto Hegel escribe que "el sistema del Derecho es el reino de la libertad efectuada, el mundo del espíritu producido a partir del espíritu él mismo como segunda naturaleza" $(1950, \S 4)$. Lo que presupone que no se accede a la comprensión genuina del Derecho si no es a partir de su inscripción en el mundo social, más precisamente, de la Sittlichkeit como "libertad consciente de sí devenida naturaleza" (Hegel 1986, $\S 513)$. Esta esfera del espíritu objetivo es una institución de sentido, entendiendo por aquella todo lo que contribuye a articular en la cultura la perspectiva individual de los sujetos en un universo normativo que les parece "natural".

Ahora bien, en lo que importa, tal determinación de la Sittlichkeit hegeliana guarda una extraordinaria proximidad con la filosofía pragmatista de Wittgenstein, en particular, y con la filosofía del lenguaje ordinario en general. En efecto, en el segundo periodo de su obra, Wittgenstein se aleja de la tesis metalingüística del Tractatus logicophilosophicus para efectuar un giro gramatical a su filosofía reivindicando el normal funcionamiento del lenguaje ordinario. Así, los problemas de la metafísica descansan sobre ilusiones gramaticales, de tal suerte que es necesario tratar terapéuticamente la filosofía para "devolver las palabras de su uso metafísico a su uso cotidiano" (Wittgenstein $2001, \S 116$ ). De este modo, el pensador austríaco se centra en demostrar que el lenguaje no es separable de la praxis y que es necesaria la integración de las reglas gramaticales en el uso cotidiano que hacen las personas del lenguaje en una comunidad. Así, la determinación normativa de una regla gramatical debe sobre todo buscarse en la aplicación práctica al interior de un contexto social donde se inscribe la acción de cada sujeto en interacción con la acción de otros según ciertas costumbres o prácticas instituidas. Es decir, según una cierta forma de vida. Como lo señala Wittgenstein, "comandar, interrogar, describir, bromear forman parte de nuestra historia natural tal como caminar, comer, beber o jugar" $(2001, \S 25)$. En los usos de los sujetos en sociedad existe una cierta convención tácita asentada en una cierta praxis que presupone ciertas normas de carácter objetivo e impersonal implícitas en los usos y costumbres que siguen los sujetos 
en un contexto social, fuera del cual carecen de valor o resultan incomprensibles. Tal como lo señala Wittgenstein en un ejemplo ilustrativo: "El coronamiento del rey es la imagen de la magnificencia y de la dignidad. Pero separado de su contexto un minuto de esta ceremonia, aquel en que depositamos la corona sobre la cabeza de rey vestido con el manto real, el oro es el metal más ordinario y su brillo parece el más vulgar" (Wittgenstein 2001, § 441). De este modo, una regla es indisociable del contexto social de su aplicación, lo que determina su alcance como su valor. En este sentido, Robert Brandom - quien ha continuado la vía del pragmatismo semántico abierta por Wittgenstein- escribe : "Las reglas no se aplican solas, ellas determinan la corrección de una acción en el contexto práctico que permite distinguir una aplicación correcta de una incorrecta de las reglas" (1994, pp. 85-86). De este modo, normas explícitas presuponen ciertas normas implícitas existentes en la praxis social.

En lo que importa, una tal aproximación ofrece un extraordinario rendimiento para una reflexión jurídica. En un sentido y en otro, entonces, el propósito es abrirse a la posibilidad de realizar una lectura postmetafísica de la filosofía del Derecho de Hegel sirviéndonos de los insumos de la filosofía social pragmatista de Wittgenstein y otros autores, al mismo tiempo que es eventualmente posible esclarecer las dificultades de la filosofía del lenguaje a partir de ciertos supuestos de la filosofía hegeliana que no han sido suficientemente considerados por la filosofía analítica, como lo es, por ejemplo, la historicidad del pensamiento.

Una propuesta de esta naturaleza permite reevaluar ciertos conceptos jurídicos a partir de esta aproximación filosófica, como lo son los conceptos de acción, causalidad, persona, o incluso libertad. En este sentido, esta propuesta pretende inscribirse en el fuerte impulso que ha recibido la filosofía del Derecho a partir de trabajos como los de Elizabeth Anscombe, que en su texto On Intentions continua el esfuerzo de Wittgenstein al practicar una verdadera "destrucción" del mito de la interioridad de la intención, para inscribirla enteramente en la exterioridad de la acción normativa (2000). Otro tanto podemos decir de la filosofía de Vincent Descombes, quien en su obra Institutions de sens (1996) ha desarrollado una filosofía social que cruza la filosofía de Wittgenstein con las ciencias sociales, elaborando ciertos presupuestos fértiles para la filosofía del derecho. Así, por ejemplo, Descombes desarrolla el concepto de una autonomía relativa según la cual el sujeto es libre y capaz de ser centro de imputación normativa de sus acciones en la medida en que ha interiorizado subjetivamente ciertas normas objetivas que señalan cómo actuar. No obstante, Descombes no omite completar tales proposiciones con la importancia que juega en el aprendizaje de una regla la confianza intersubjetiva depositada en una actuación correcta. En lo que importa, no se trata solo de considerar el Derecho como un dispositivo normativo de producción de subjetividad, sino que reconocer en el sujeto una capacidad para ser centro irreductible de imputación de sus acciones que pasa necesariamente por una interacción social que implica-igualmenteuna cierta determinación objetiva de su personalidad y de sus capacidades. Las normas -irreductibles a los sujetos- están vivas en la medida en que los sujetos se sirven de ellas. Tal es el dominio de una libertad que es a la vez objetiva y subjetiva.

En este orden de consideraciones es inevitable no ver en este proyecto una cierta revalorización del pensamiento juvenil de Marx y su reivindicación de la praxis en el 
texto de La ideología alemana. ¿Acaso Marx no insiste en sus trabajos más filosóficos que no es la conciencia la que determina la vida, sino la vida a la conciencia? Inspiración marxista doblemente, porque un proyecto de este tipo se inscribe en un holismo ético con innegables consecuencias políticas frente a un individualismo ético liberal que presupone la disolución del nexo entre el hombre y las instituciones (Ehrenberg 2011).

\section{Algunas consideraciones finales}

No obstante, una propuesta como esta tiene a lo menos dos dificultades. Por una parte, la necesidad de hacerse cargo de una cierta filosofía contemporánea de inspiración antiinstitucionalista, que a partir de Foucault se aproxima de forma radicalmente crítica a la dimensión objetiva del Derecho. O bien, de forma más moderada, una filosofía crítica que intenta comprender el Derecho a partir de un sustrato sub-institucionalista como es el caso de la teoría del reconocimiento de Axel Honneth y la tercera generación de la teoría crítica, que busca hacer un lugar al Derecho dentro de una economía del reconocimiento intersubjetivo, teniendo en vista justamente las críticas institucionalistas de Foucault. Cuestión que tiene que ver -por cierto- con el esfuerzo de la teoría crítica por resolver las fracturas sociales en la modernidad capitalista, lo que pasa necesariamente por pronunciarse sobre el Derecho como institución o agente socializador (Durkheim 2013).

Pero las dificultades también asoman desde el punto de vista de la obra hegeliana. En efecto, esta propuesta que busca reivindicar el rol del espíritu objetivo en Hegel, ¿tiene a la vista acaso que el espíritu objetivo está asociado a la infraestructura lógicametafísica del Sistema hegeliano? Hegel nos da a entender, en la inserción de la Enciclopedia con la Lógica, que el espíritu objetivo -y en consecuencia el Derechoson un momento en el movimiento del Absoluto. Incluso Marx mismo señala en su crítica del derecho político hegeliano, que la filosofía del derecho no es más que un paréntesis de la Lógica... No obstante, esta aproximación está sujeta a una hipoteca metafísica de la que hay que hacerse cargo antes de entrar de forma decidida en la Lógica hegeliana sin caer en una metafísica especulativa, lo cual pasa por evaluar si la parte metafísica de la obra hegeliana es el conjunto de la Lógica o, más precisamente, el pasaje al espíritu absoluto...cuestión que aquí no está resuelta. ¿Acaso Marx, que se inscribe de forma resuelta en la salida de la metafísica, no califica a la Lógica como el núcleo racional de la dialéctica revolucionaria en epílogo de El Capital (2004)? ${ }^{5}$ En fin, tantos desafíos como los que naturalmente forman parte de hacer filosofía. Sobre todo si se trata de Hegel.

$5 \quad$ Cfr. Engels, Friedrich, Die Entwicklung des Sozialismus von der Utopie zur Wissenschaft, Berlin: Dietz Verlag, 1980. En el mismo sentido, Espinoza Lolas, 2016. Lenin lo menciona explícitamente: "pese a que Marx nunca escribió su proyectado tratado breve sobre la dialéctica, nos dejó sin embargo El Capital, que es la aplicación de la dialéctica materialista al campo de los fenómenos económicos". Lenin, 1980. 


\section{Referencias bibliográficas}

Anscombe, Elizabeth (2000), On Intentions. Cambridge: Harvard University Press.

Bourgeois, Bernard (1986), Le Droit naturel de Hegel. Paris: Vrin.

Brandom, Robert (1994), Making It Explicit: Reasoning, Representing, and Discursive Commitment. Cambridge: Harvard University Press.

Descombes, Vincent (1996), Institutions de sens. Paris: Minuit.

Durkheim, Emile (2013), De la division du travail social. Paris: PUF.

Ehrenberg, Alain (2011), Le culte de la performance. Paris: Fayard/Pluriel.

Engels, Friedrich (1980), Die Entwicklung des Sozialismus von der Utopie zur Wissenschaft, Berlin: Dietz Verlag.

Espinoza Lolas, Ricardo (2016), Hegel y las nuevas lógicas del mundo y del Estado, Madrid: Akal.

Hegel, GWF (1986), Enzyklopädie der philosophischen Wissenschaften 1817-1830. Werke in zwanzig Bänden, Bd.10. Frankfurt a.M.: Suhrkamp.

(1968), Glauben und Wissen. Gesammelte Werke, Bd. 4. Hamburg: Felix Meiner Verlag.

(1950), Grundlinien der Philosophie des Rechts, Sämtliche Werke, Bd. 7, hgg. v. H. Glockner. Stuttgart: Fromann Verlag.

(1967), Über das Wesen der philosophischen Kritik überhaupt und ihr Verhältnis zum gegenwärtigen Zustand der Philosophie insbesondere. Hildesheim: Georg Olms Verlagsbuchhandlung.

(1969-1971), Vorlesungen über die Geschichte der Philosophie. Werke in zwanzig Bänden, Bd.10. Frankfurt a.M.: Suhrkamp.

(1955), Vorlesungen über Naturrecht und Staatswissenschaft, Heidelberg 1817-1818 (mit Nachträgen aus den Vorlesung 1818/1819). Hamburg: Meiner Verlag.

Hobbes, Thomas (2000), Leviathan. Trad. Marinet. Paris: Gallimard.

Kant, Immanuel (1968), Metaphysik der Sitten. Kants Werke Akademie Textausgabe, vol.VI. Berlin: De Gruyter Verlag.

Kelsen, Hans (1993), Teoría pura del Derecho, Ciudad de México: Porrúa, pp. 194195.

Lenin, Vladimir I. (1980), Obras completas. México: Ediciones de Cultura Popular/ Akal, 1980, p. 305.

Marx, Karl (2004), El Capital. Libro primero. Buenos Aires: Siglo XXI editores.

Nohl, Hermann (1907), Hegels theologische Jugendschriften nach den Handschriften. Tubingen: Mohr.

Pinkard, Terry (1996), Hegel's Phenomenology: the sociality of reason. Cambridge: Cambridge University Press.

Pippin, Robert (1989), Hegel's idealism: The satisfaction of self-consciousness. Cambridge: Cambridge University Press. 
Rorty, Richard (1991), Objectivity, Relativism and Truth: Philosophical Papers I. Cambridge: Cambridge University Press.

Rousseau, Jean Jacques (1821), Émile ou de l'éducation. Trad. Marchena. Madrid: Alban.

Pufendorf, Samuel von (1989), Droit de la nature et des gens. Trad. Jean Barbeyrac. Caen: Centre de philosophie politique et juridique.

Spinoza, Baruch (2005), Ética demostrada según el orden geométrico. Edición y traducción de Atilano Domínguez. Madrid: Editorial Trotta.

Valenzuela Feijóo, José Carlos (2006). Libertad y Razón: Rousseau, Hegel, Marx. Santiago: LOM Ediciones.

Wittgenstein, Ludwig (2001), Philosophische Untersuchungen. Kritisch-genetische Edition. Frankfurt a.M.: Wissenschaftliche Buchgesellschaft. 